\title{
The chemical fingerprints of the thin and the thick disk
}

\author{
Sofia Feltzing ${ }^{1}$, Sally Oey ${ }^{2}$ and Thomas Bensby ${ }^{3}$ \\ ${ }^{1}$ Lund Observatory, \\ Box 43, SE-221 00 Lund, Sweden \\ email: sofia@astro.lu.se \\ ${ }^{2}$ University of Michigan \\ 830 Dennison Building, Ann Arbor, MI, USA 48109-1042, USA \\ ${ }^{3}$ European Southern Observatory \\ Alonso de Cordova 3107, Vitacura, Casilla 19001, Santiago 19, Chile \\ email: tbensby@eso.org
}

\begin{abstract}
The past history and origin of the different Galactic stellar populations are manifested in their different chemical abundance patterns. We obtained new elemental abundances for $553 \mathrm{~F}$ and $\mathrm{G}$ dwarf stars, to more accurately quantify these patterns for the thin and thick disks. However, the exact definition of disk membership is not straightforward. Stars that have a high likelihood of belonging to the thin disk show different abundance patterns from those for the thick disk. In contrast, we show that stars for the Hercules Stream do not show unique abundance patterns, but rather follow those of the thin and thick disks. This strongly suggests that the Hercules Stream is a feature induced by internal dynamics within the Galaxy rather than the remnant of an accreted satellite.
\end{abstract}

Keywords. stars: abundances, stars: kinematics, Galaxy: abundances, Galaxy: disk, Galaxy: formation

\section{Chemical fingerprints of the Milky Way stellar disks}

That the Milky Way has two stellar disks became clear when the existence of the thick disk was confirmed through star counts (Gilmore \& Reid 1983). The existence of the two stellar populations in the Milky Way disks with partly overlapping properties has since been confirmed in a number of studies, notably those on elemental abundances and ages (e.g., Gratton et al. 2003, Soubiran \& Girard 2005, Reddy et al. 2003, Reddy et al. 2006). In general, the thick disk is observed to be older and less metal-rich than the thin disk.

Of particular concern to us in the context of galactic chemical evolution is the fact that these two disk components appear to have distinct abundance patterns. Here we will, as an example, discuss two studies of elemental abundances in $F$ and $G$ dwarf stars in the solar neighbourhood: Fuhrmann (2008) and Bensby et al. (2004). These two studies nicely illustrate some of the best established properties of the thin and the thick disks.

Fuhrmann (2008) studied the trends for magnesium relative to iron and found that his volume-limited sample $(d<25 \mathrm{pc})$ of late $\mathrm{F}$ and early $\mathrm{G}$ dwarf stars with respect to the elemental abundances split into two major groups. One of these shows an overabundance of $\mathrm{Mg}$ relative to $\mathrm{Fe}$ of about +0.4 dex, presenting a plateau that extends from an $\mathrm{Fe}$ abundance of -1 to -0.3 dex. Fuhrmann associates these stars with the Milky Way thick disk. The other major group consists of stars that are less enhanced in $\mathrm{Mg}$ relative to $\mathrm{Fe}$, and these stars also show a shallow decline in $[\mathrm{Mg} / \mathrm{Fe}]$ as $[\mathrm{Fe} / \mathrm{H}]$ increases. Fuhrmann also found that the stars with less enhancement of $\mathrm{Mg}$ were younger than the stars with 

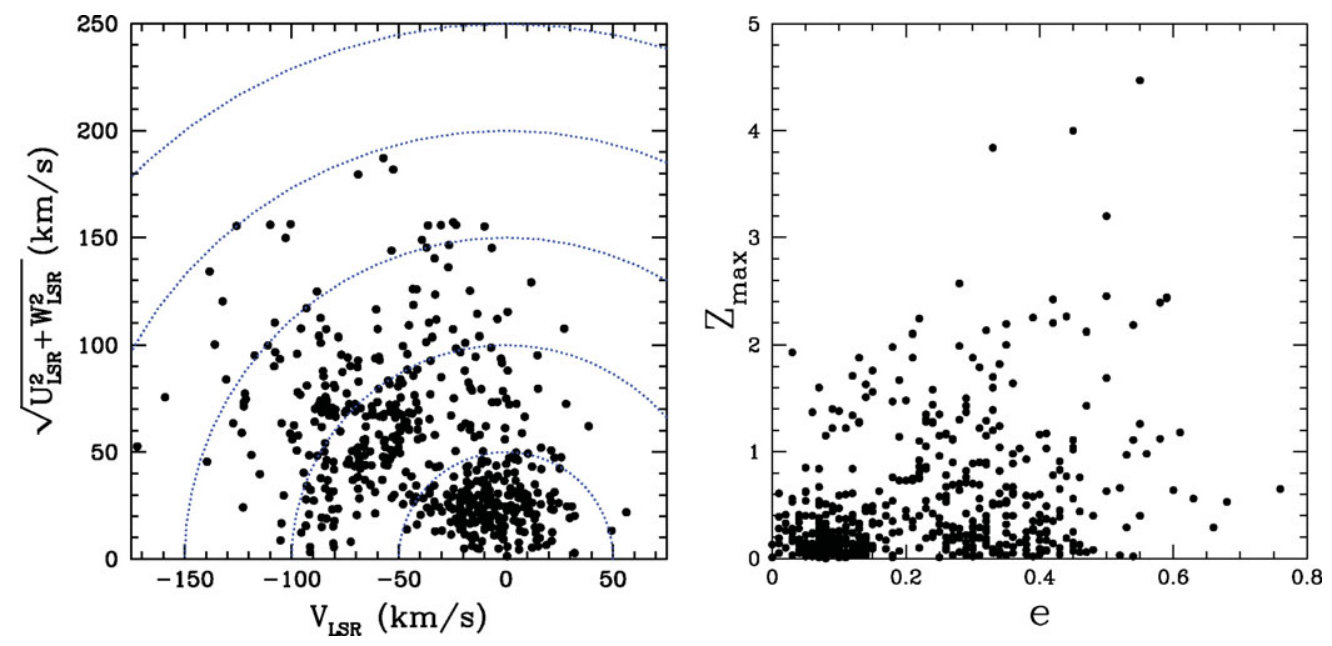

Figure 1. Left hand panel: Toomre diagram showing the kinematic properties for the sub-sample of five hundred stars. Right hand panel: Two orbital parameters, maximum height above the Galactic plane in $\mathrm{kpc}\left(Z_{\max }\right)$ and ellipticity of the orbit $(e)$ for the same stars. The orbital parameters are taken from the calculations in Nordström et al. (2004).

higher Mg enhancement. He identifies these stars with the thin disk. Stars that do not fall onto either of the two trends were dubbed "transition" stars by Fuhrmann.

Bensby et al. (2004), on the other hand, employed kinematic criteria to define two samples of $\mathrm{F}$ and $\mathrm{G}$ dwarf stars: one in which the stars have a much higher probability to be thin than thick disk stars, and one in which they have a much higher probability to be thick than thin disk stars. For a full description of how the stars were selected see Bensby et al. (2003). For these stars, we obtained oxygen abundances from the forbidden line at $630.0 \mathrm{~nm}$. Also for these samples, a clear distinction was found between the abundance trends traced by the thin and thick disk stars, respectively, such that the thick disk stars are more enhanced in $\mathrm{O}$ relative to $\mathrm{Fe}$ than the thin disk is.

Hence, we may conclude that at least one of the chemical fingerprints for the thin and the thick disks is this difference in enhancement in $\alpha$-elements relative to iron. Furthermore, this trend appears to be quite insensitive to the actual definition of thin vs thick disk stars: both a volume-complete, as well as kinematically selected samples, show the same overall picture, even though the details might differ.

However, even though it appears to be reasonably easy to kinematically distinguish the two major components of the stellar disk, the thin and the thick disk, it is clear that the stars in the solar neighbourhood are not smoothly distributed in velocity space, (Nordström et al. 2004). Indeed, several streams and so-called moving groups have been identified. The origin of these kinematic groupings is, however, not altogether clear; are they dissolved stellar clusters, or accreted satellites, or do they result from various dynamical process such as resonances with the Galactic bar (e.g. Dehnen 2000). Studies of the chemical fingerprints of such kinematic structures will help to settle these questions, as we show below (see also Freeman \& Bland-Hawthorn 2002).

\section{A new sample of stars}

We have now extended our studies to include about $900 \mathrm{~F}$ and $\mathrm{G}$ dwarf stars. The full sample consists of several sub-samples that were each defined to study a particular question, e.g., the question of how metal-rich the thick disk can be (Bensby et al. 2007b). 


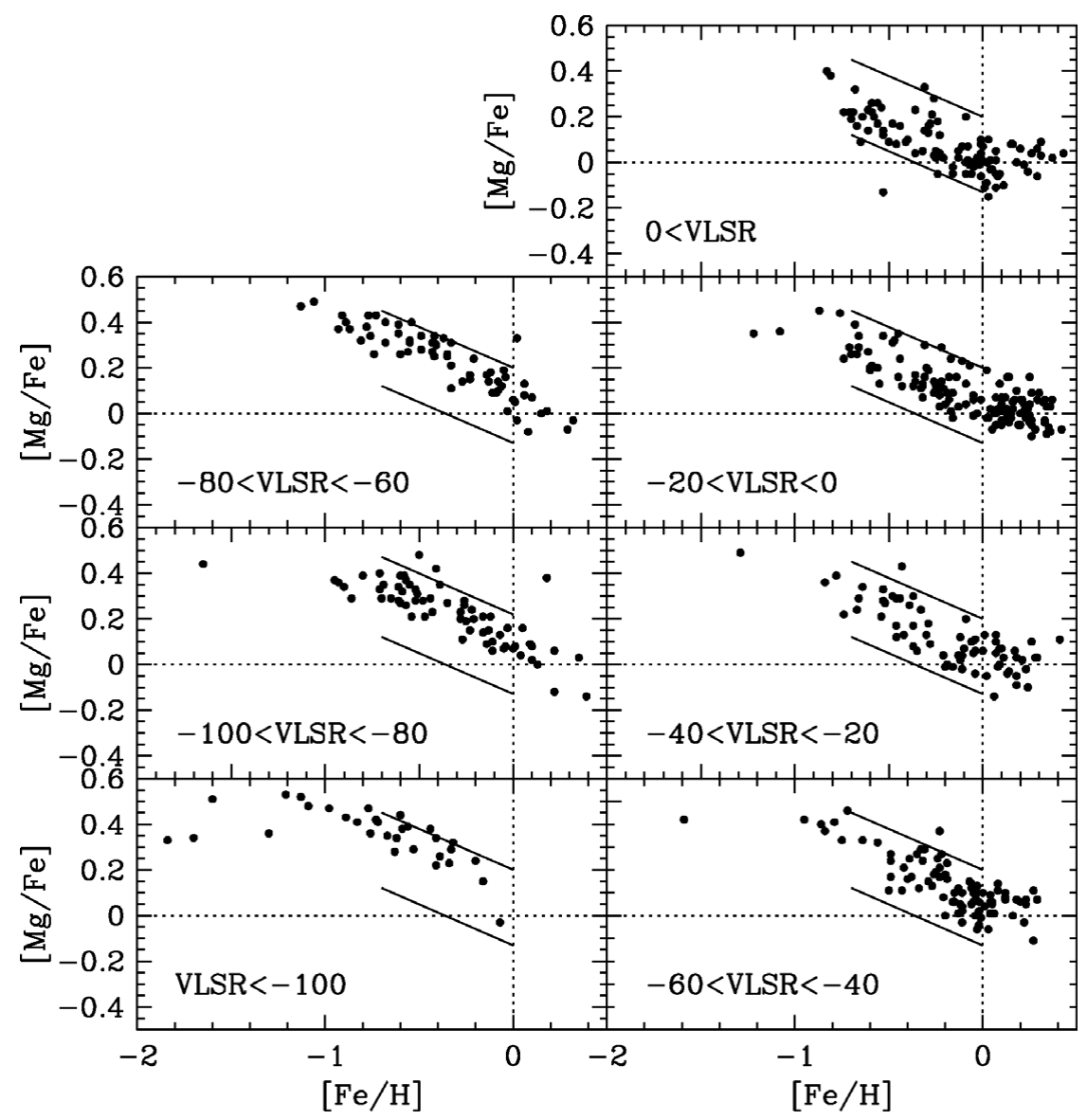

Figure 2. $[\mathrm{Mg} / \mathrm{Fe}]$ vs $[\mathrm{Fe} / \mathrm{H}]$ for samples with different $V_{\mathrm{LSR}}$ velocities, as indicated. The solid lines are to guide the eye and have been fitted to trace the upper and lower boundaries of the full sample.

For these stars we have obtained high-resolution, high S/N spectra using several spectrographs (Bensby et al. in prep.). Normally, $\mathrm{S} / \mathrm{N}$ is above 250 and the resolution, for a significant fraction of the stars 60,000 or higher. In this contribution we present the first results for a subset of $\sim 500$ of these stars. The elemental abundances and stellar parameters are derived using a methodology based on that used in Bensby et al. (2003). We base our $\log g$ and age determinations on the Hipparcos parallaxes, and the effective temperatures on excitation balance (Bensby et al., in prep.). However, we now include enhancement of $\alpha$-elements in the model atmospheres and have developed a more automated approach to the determination of stellar parameters based on a large grid of model atmospheres. The kinematic properties and some orbital parameters of this sub-sample are shown in Fig. 1. The orbital parameters are taken from Nordström et al. (2004).

\subsection{Elemental abundances and kinematics are intimately connected}

Here, we investigate how the elemental abundance trends change as a function of $V_{\mathrm{LSR}}$, where $V_{\mathrm{LSR}}$ is the velocity in the direction of rotation around the centre of the Galaxy, $U_{\text {LSR }}$ is the radial velocity, and $W_{\text {LSR }}$ the vertical velocity. Fig. 1 shows the distribution of velocities for our sample. 


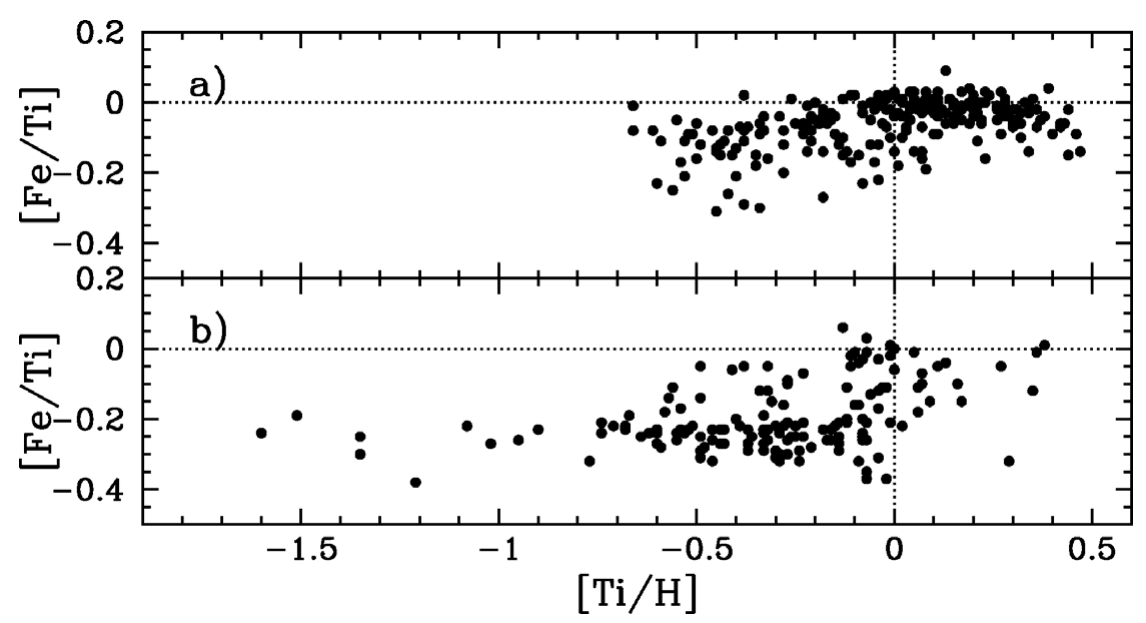

Figure 3. a) $[\mathrm{Fe} / \mathrm{Ti}]$ vs. $[\mathrm{Ti} / \mathrm{H}]$ for stars ten times more likely to be thin than thick disk stars.

b) $[\mathrm{Fe} / \mathrm{Ti}]$ vs. $[\mathrm{Ti} / \mathrm{H}]$ for stars ten times more likely to be thick than thin disk stars.

Figure 2 shows how the elemental abundances, in this case $[\mathrm{Mg} / \mathrm{Fe}]$ as a function of $[\mathrm{Fe} / \mathrm{H}]$, change as a function of $V_{\mathrm{LSR}}$. Clearly, there is a change in the trend as we move to lower velocities. In this simplistic plot we see, around $0 \mathrm{~km} \mathrm{~s}^{-1}$, the trend associated with the local thin disk. However, as we proceed to lower velocities, $[\mathrm{Mg} / \mathrm{Fe}]$ becomes progressively more enhanced. It is interesting to note that for essentially all velocity bins, we are sampling a large range of $[\mathrm{Fe} / \mathrm{H}]$, reaching up to solar metallicities and beyond, in all but the last bin. For the first three velocity bins $\left(0<V_{\mathrm{LSR}},-20<V_{\mathrm{LSR}}<0\right.$, $\left.-40<V_{\mathrm{LSR}}<-20\right)$, all stars with $[\mathrm{Fe} / \mathrm{H}]>0$ have $e<0.15$, and they are therefore not likely candidates for stars that have migrated from the inner disk.

It is interesting to note that in, e.g., the bin with $-80<V_{\mathrm{LSR}}<-60$, the metal-rich stars are more elevated in their $[\mathrm{Mg} / \mathrm{Fe}]$. Here, most stars with $[\mathrm{Fe} / \mathrm{H}]>0$ have orbits with higher ellipticity, i.e., they are probing the disk interior to the solar circle. This could thus potentially be a signature of a more rapid star formation process, leading to higher levels of $[\mathrm{Mg} / \mathrm{Fe}]$, in the inner disk. However, this interpretation is tentative and requires more investigation.

\subsection{Abundance trends in kinematically defined samples of the thin and the thick disk}

We now proceed to look at the thin and thick disk defined using the kinematic criteria developed by Bensby et al. (2003). For the thick disk, we chose stars that are ten times more likely to belong to the thick than thin disk, and for the thin disk stars, ones that are ten times more likely to belong to the thin than thick disk. Figure 3 shows the resulting abundance trends for $[\mathrm{Fe} / \mathrm{Ti}]$ vs. $[\mathrm{Ti} / \mathrm{H}]$. Ti is here preferred as the reference element instead of the commonly used Fe, as Ti is mainly produced in SN II, while Fe is produced in both SN II and SN Ia.

The two stellar samples show different abundance trends. The thin disk sample has a sharp, upper envelope that defines a gentle curve. The thick disk sample has a clear "bottom", a floor that defines the highest level of $\alpha$-enhancement in the stars. This plateau ranges from $[\mathrm{Ti} / \mathrm{H}]=-1.5$ to solar $\mathrm{Ti}$ values. At the solar value $[\mathrm{Fe} / \mathrm{Ti}]$ increases rapidly. 


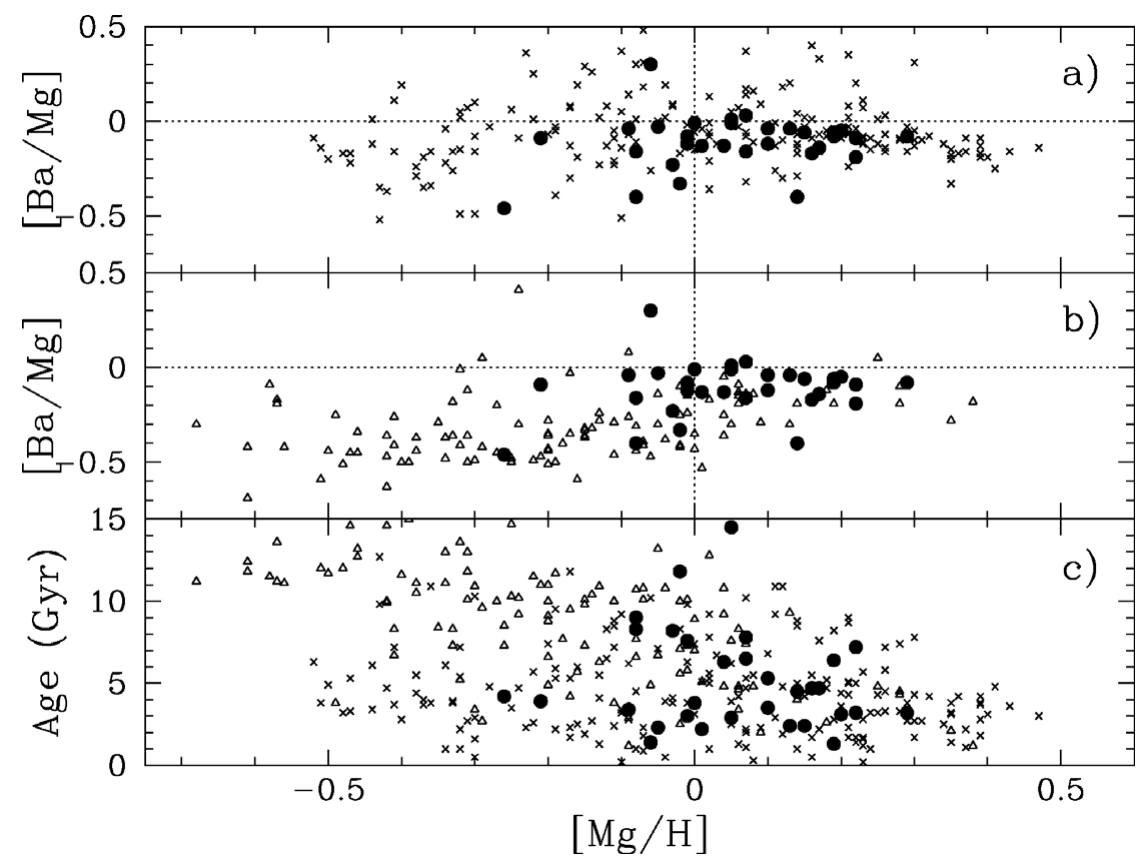

Figure 4. Elemental abundances and ages for the Hercules stream. We only show data for stars that have internal uncertainties in the age determinations of less than $3 \mathrm{Gyr}$. a) $[\mathrm{Ba} / \mathrm{Mg}] \mathrm{vs}$ $[\mathrm{Mg} / \mathrm{H}]$ for thin disk $(\times)$ and Hercules stars $(\bullet)$. b) $[\mathrm{Ba} / \mathrm{Mg}]$ vs $[\mathrm{Mg} / \mathrm{H}]$ for thick disk $(\triangle)$ and Hercules stars $(\bullet)$. c) Stellar ages as a function of $[\mathrm{Mg} / \mathrm{H}]$. Symbols as in $a$ ) and $b$ ).

\section{The Hercules stream}

The existence of unique abundance patterns for the thin and thick disks allows us to test whether local kinematic features are comprised of stars that are members of the thin and thick disks, or whether they might be remnants of accreted satellite galaxies. Famaey et al. (2005) found that the Hercules stream of stars makes up about $6 \%$ of the stars in the solar neighbourhood, and that they have a net drift of $\sim 40 \mathrm{~km} \mathrm{~s}^{-1}$ directed radially away from the Galactic centre. The stream is also lagging behind the Local Standard of Rest by about the same amount as the thick disk. We used this kinematic information to isolate $60 \mathrm{~F}$ and $\mathrm{G}$ dwarf stars as likely members of the Hercules stream. For these stars, we obtained high-resolution $(R \sim 65,000)$, high $\mathrm{S} / \mathrm{N}(\geqslant 250)$ spectra with the MIKE spectrograph on Magellan. The selection of stars, data reduction and subsequent abundance analysis are detailed in Bensby et al. (2007a) and Feltzing et al. (in prep.).

We find that the Hercules stream does not consist of stars with a unique metallicity, nor with any other unique feature. Instead, the members of the Hercules stream fall on the trends of both the thin and thick disks, indicating that they are only members of the underlying general disk populations. Dehnen (2000) indeed showed that the Hercules stream might be the result of the dynamical interaction between the local disk and the bar. Our data seem to support this model.

In fact, it appears more and more likely that the majority of the moving groups and stellar streams do not originate from a single stellar cluster or an accreted dwarf galaxy, but rather are the results of dynamical processes within the disk itself. A nice illustration of this is given by the study of the Arcturus group by Williams (see these proceedings). In fact, so far it is only the HR1614 moving group that has shown itself to be a single 
age, single abundance population, indicating that all stars come from the same cluster (De Silva et al. 2007).

\section{Discussion and Conclusions}

We find that for our enlarged, kinematically defined stellar sample, the thin and the thick disks also show distinct abundance trends. While these trends are fairly robust to statistically defined selection criteria, the distinctions are somewhat less clear than in the previous, smaller samples that we have studied. Our interpretation is that the disk is a complex entity and that clear trends probably can never be obtained when we use statistically defined selection criteria. Our criteria to define the thin disk tend to produce the most consistent abundance trends. Obviously, the thin disk is the easiest population to define both kinematically and spatially. The thick disk, represented by less than $15 \%$ the number of thin disk stars in our sample (see, e.g., Árnadóttir et al. 2008), has less well-defined properties at present. However, it is clear that the thick disk is more enhanced in $\alpha$-elements than the thin disk at a given metallicity. It might prove fruitful to study the in situ thick disk stars in order to find a clean signature of the thick disk. We further exploited the thin and thick disk abundance patterns to test whether the Hercules Stream has an extragalactic origin, and its normal abundance patterns suggest that it does not.

In summary, the different Galactic stellar populations present well-defined, differentiated element abundance patterns that offer fundamental diagnostics of their origin. These data set strong constraints on Galactic chemical evolution models for the history of the Milky Way.

\section{References}

Árnadóttir, A., Feltzing, S., \& Lundström, I 2008, arXiv:0807.1665

Bensby, T., Feltzing, S., \& Lundström, I. 2003, A\&A, 410, 527

Bensby, T., Feltzing, S., \& Lundström, I. 2004, A\&A, 415, 155

Bensby, T., Oey, M. S., Feltzing, S. \& Gustafsson, B. 2007a, ApJL, 655, 89

Bensby, T., Zenn, A. R., Oey, M. S., \& Feltzing, S. 2007b, ApJL, 663, 13

Dehnen, W. 2000, AJ, 119, 800

De Silva, G. M., Freeman, K. C., Asplund, M., Bland-Hawthorn, J., Bessel, M. S., \& Collet, R. 2007, AJ, 133, 1161

Famaey, B., Jorissen, A., Luri, X., Mayor, M., Udry, S., Dejonghe, H., \& Turon, C. 2005, A\&A, 430,165

Freeman, K. \& Bland-Hawthorn, J. 2002, ARA\&A, 40, 487

Fuhrmann, K. 2008, MNRAS, 384, 173

Gilmore, G. \& Reid, N. 1983, MNRAS, 202, 1025

Gratton, R. G., Carretta, E., Desidera, S., Lucatello, S., Mazzei, P., \& Barbieri, M. 2003, A\&A, 406, 131

Nordström, B., Mayor, M., Andersen, J., Holmberg, J., Pont, F., Jørgensen, B. R., Olsen, E. H., Udry, S., \& Mowlavi, N. 2004, A\&SA, 418, 989

Reddy, B. E., Lambert, D. L., \& Allende Prieto, C. 2006, MNRAS, 367, 1329

Reddy, B. E., Tomkin, J., Lambert, D. L., \& Allende Prieto, C. 2003, MNRAS, 340, 304

Soubiran, C. \& Girard, P. 2005, A\& A, 438, 139 